\title{
Un proceso de diálogo multiactor para la paz en el País Vasco
}

\author{
A multi-stakeholder dialogue process \\ for peace in the Basque Country
}

\author{
Xavier Mínguez-Alcaide / xavier.minguez@ehu.eus \\ Grupo de Investigación Gune Irekiak, España
}

\begin{abstract}
The article presents a dialogue process within the framework of the Basque Country conflict. Taking the proposals of systemic conflict transformation approach, a scene of dialogue was designed to generate consensuses on the fundamental issues that involves peace in Basque Country. After exposing the study's theoretical foundation, the article explains the elements and phases of process of ongoing dialogue. Later, it presents consensuses obtained on the questions worked, that is, reconciliation and coexistence, memory and historical narrative, the political dimension of conflict, citizen participation for peace, victims and prisoners. Finally, the paper presents recommendations for peace in Basque Country, as the participation of all stakeholders, the adoption of the human rights as common floor for peace, the disarming of ETA and the transformation of penitentiary and counterterrorism policy.
\end{abstract}

Key words: Basque Country, systemic conflict transformation, peacebuilding, multistakeholder dialogue, Open Space Technology.

Resumen: El artículo presenta un proceso de diálogo en el marco del conflicto del País Vasco. Desde una aproximación sistémica a la transformación de conflictos, se diseñó una escena de diálogo para generar consensos sobre las cuestiones fundamentales para la paz en el País Vasco. Tras la fundamentación teórica del estudio, se explica los elementos y fases del proceso de diálogo sostenido. Posteriormente, se presentan los consensos alcanzados sobre las cuestiones trabajadas: reconciliación y convivencia, memoria y narrativa histórica, la dimensión política del conflicto, la participación ciudadana para la paz, víctimas y presos. Finalmente, el artículo presenta recomendaciones para la paz en el País Vasco, como la participación de todos los grupos de interés, la adopción de los derechos humanos como núcleo para la paz, el desarme de ETA y la transformación de la política penitenciaria y antiterrorista.

Palabras clave: País Vasco, transformación sistémica de conflictos, construcción de paz, diálogo multiactor, Open Space Technology. 


\section{Introducción}

Desde una perspectiva académica, el abordaje de la paz y los conflictos profundos es muy prolífico y heterogéneo. De manera muy resumida, Gawerc (2006) afirma que nos encontramos con propuestas estructuralistas que se centran básicamente en cuestiones políticas, y otras de tipo psicosocial que ponen el foco en las necesidades humanas básicas, las relaciones humanas y en los elementos psicológicos subyacentes a los conflictos profundos.

Desde la óptica sistémica de la transformación de conflictos, tanto las cuestiones estructurales como las psicosociales tienen un peso específico, ya que los conflictos se conciben como sistemas complejos, es decir, como una red de elementos psicológicos, sociales y estructurales relacionados entre sí, siendo las dinámicas relacionales entre elementos el motor que mantiene el sistema/conflicto en equilibrio, y por lo tanto, lo que determina que se perpetúe (Coleman, 2006 y 2012; Körpen et al., 2011).

Por ello, la transformación del conflicto y la construcción de paz requieren de romper las dinámicas relacionales entre los elementos centrales del sistema/ conflicto -denominados atractores-, ya sean de naturaleza psicosocial o estructural, y con ello romper el equilibrio del sistema/conflicto para poder transformarlo (Vallacher et al., 2010). Coleman et al. (2007: 14) dicen que "lo que se mantiene constante y funciona para perpetuar el conflicto son las dinámicas que definen las relaciones entre los mecanismos psicológicos y sociales dentro y entre los individuos y los grupos".

Los atractores del sistema/conflicto son estados o patrones de cambio hacia los que éste se mueve (Novak et al., 2012). De manera concreta, los atractores son factores psicológicos, sociales y estructurales muy estables y de gran consistencia, que mantienen relaciones muy fuertes entre sí y con otros elementos del sistema/conflicto. Ejemplos de atractor en conflictos profundos pueden ser los balances asimétricos de poder, historias de dominación étnico-cultural o político-económica, relaciones suma-cero entre objetivos grupales, niveles elevados de hostilidad intergrupal, normatividades grupales fuertes, climas emocionales negativos de odio y miedo, o patrones de pensamiento y percepción rígidos, simples y polarizados.

Desde esta óptica, afrontar los conflictos requiere de romper las dinámicas sistémicas que mantienen la robustez de los atractores del conflicto, y desarrollar atractores de paz para generar nuevos equilibrios. Para la emergencia de estos atractores de paz se propone generar iniciativas con objetivos diversos, teniendo en cuenta el largo y el corto plazo - cese de la violencia, logro de acuerdos de paz, reconstrucción del tejido social y reconciliación-, y que 
impliquen a múltiples grupos de las élites, los niveles sociales intermedios y el conjunto de la ciudadanía (Novak et al., 2012). Entre las iniciativas para generar atractores de paz, los autores señalan la posibilidad de generar redes de acción a partir del diálogo entre personas con diferentes puntos de vista, y aplicar procedimientos basados en la democracia deliberativa.

La creación de espacios de participación y diálogo se considera fundamental en toda aproximación a la construcción de paz. Desde el enfoque sistémico, se enfatiza la necesidad de generar espacios y procesos de participación, interacción y diálogo con personas pertenecientes a diversos grupos o sectores de interés que son clave para la paz (Duran, 2013; Unger y Wills: 2006; Wills et al., 2006). Del mismo modo que Lederach (1998) afirma la necesidad de establecer puentes horizontales y verticales para la paz, haciendo que se crucen las líneas del conflicto tanto en los mismos estratos sociales como entre ellos, Wills et al. (2006) afirman la necesidad de intervenciones inclusivas multipartes, donde estén presentes miembros de grupos críticos y sectores sociales diferentes, como los partidos políticos, los medios de comunicación, la Iglesia, la educación, o las ONG y grupos de derechos humanos locales.

Siguiendo la propuesta sistémica de transformación de conflictos para construir un escenario de paz sostenible, y haciendo énfasis en la participación de múltiples actores con relevancia social, el presente estudio se planteó con los objetivos básicos de, por un lado, favorecer el desarrollo de relaciones de confianza entre personas jóvenes de partidos políticos, sindicatos, la universidad, y movimientos pacifistas y de derechos humanos; y por otro, llegar a consensos básicos sobre los desafíos centrales de la construcción de la paz en el País Vasco que sirvieran de recomendaciones a seguir.

\section{Caso de estudio. El conflicto y la construcción de paz en el País Vasco}

El caso del País Vasco puede considerarse un conflicto etnonacional complejo, ya que presenta dimensiones características de este tipo de conflictos (Gurr, 2000; Horowitz, 1985). Siguiendo una óptica psicosocial compleja, podemos inferir que los atractores centrales del caso vasco, es decir, aquellos elementos que mantienen el conflicto en equilibrio, están vinculados con una interdependencia negativa en la construcción de las identidades nacionales vasca y española (y francesa), una contradicción nacional en términos políticos y de lucha por el poder y la soberanía nacional sobre los territorios vascos en España y Francia, y la utilización de diferentes formas de violencia política por parte 
del Movimiento Vasco de Liberación Nacional $\left(\mathrm{MLNV}^{1}\right)$ y de agentes directa o indirectamente vinculados al Estado Español, como las Fuerzas de Seguridad del Estado, o grupos parapoliciales y de extrema derecha como los Grupos Antiterroristas de Libertación (GAL), el Batallón Vasco Español (BVE) o la Alianza Apostólica Anticomunista (Triple A), con sus lógicas de legitimación social y sus consecuencias a nivel social.

Estos anclajes del conflicto aparecen vinculados con una serie de procesos de corte psicosocial, como son un impacto negativo sobre la Necesidades Humanas Básicas de tipo psicosocial -como el desarrollo de las identidades colectivas, la seguridad, el sentido de justicia o la percepción del control sobre el propio destino-, o elementos característicos de las identidades colectivas polarizadas, como patrones de percepción y pensamiento rígidos, simplificadores y polarizados, actitudes confrontacionales y evitativas del conflicto o narrativas históricas contradictorias (Mínguez-Alcaide, 2013).

Teniendo en cuenta la borrosidad como herramienta de análisis de la complejidad, el conflicto vasco se nos muestra como claro ejemplo de dicha borrosidad. Ello lo vemos en una estructura social muy compleja, con una importante parte de la sociedad que, de manera creciente, ha mantenido posiciones más o menos intermedias en el conflicto, expresada en su contrariedad tanto hacia la violencia de Euskadi Ta Askatasuna (ETA) como hacia la política antiterrorista del Estado Español, en un ambiente de fuerte presión a la polarización por parte de los polos del conflicto (el MLNV y el Estado Español).

Ello también se ve reflejado en la pluralidad identitaria de la sociedad vasca, pues en los territorios vascos conviven diferentes formas de identificación con lo vasco y lo español (y francés), que siguen un continuo desde las identidades más monolíticas de marcado carácter étnico-cultural, construidas con base en una percepción de suma-cero, hasta las más abiertas donde se vislumbran diferentes formas de compatibilidad entre las mismas.

Desde un punto de vista histórico, se han sucedido diferentes etapas en los siglos XIX y XX, donde la disputa por la soberanía y la administración de los territorios vascos ha sido un elemento central (Apalategi, 2006). Así, el uso de la violencia política con el objetivo de sustentar el poder sobre los territorios vascos es un elemento presente en la historia del País Vasco, tanto en los siglos XIX y XX, como en la primera década del siglo XXI. Para la comprensión del conflicto vasco desde una perspectiva histórica reciente, es imprescindible tener en cuenta el periodo de la dictadura del general Franco

1 Movimiento Vasco de Liberación Nacional, que incluye a ETA como organización armada, partidos políticos Herri Batasuna o Batasuna (ilegalizados) y Sortu (legal), y otras estructuras sociales. 
(1939-1975), y sobre todo el pasado más reciente de transición y construcción de un sistema democrático liberal, a partir de 1975.

La manifestación contemporánea del conflicto vasco está vinculada al MLNV y la actividad armada de ETA. ETA nace en 1959 como continuación de la organización estudiantil de carácter nacionalista Ekin (AlvarezEnparantza, 1997) y desarrolla su ideario influida por la ideología de los movimientos revolucionarios y de liberación nacional de los años cincuentasesenta (Lizarralde, 2012).

Tras 50 años de existencia, con más de 850 muertes perpetradas, y habiendo condicionado la vida social y política vasca y española desde su creación, el 10 de enero de 2011 ETA envía un video y un comunicado al diario Gara, en el cual declara un alto al fuego permanente y de carácter general, que puede ser verificado por la comunidad internacional (ETA, 2011a). Posteriormente, tras la Conferencia Internacional de Paz de San Sebastián, celebrada el 17 de octubre de 2011, conocida como la Conferencia de Aiete, el 20 de octubre de 2011, ETA declara el cese definitivo a su actividad armada (ETA, 2011b).

\section{Método. Un proceso de diálogo para la paz en el País Vasco}

El objetivo del presente estudio era generar un espacio de confianza y diálogo sostenido, donde personas jóvenes de actores claves para la paz -partidos políticos, sindicatos, movimientos pacifistas y de derechos humanos, medios de comunicación y la universidad-pudieran tratar los desafíos centrales que supone construir la paz en el País Vasco, llegando a consensos en la medida de lo posible, y haciendo que éstos sirvieran de recomendaciones para apoyar el proceso de construcción de paz. El proceso de diálogo continuado se desarrolló entre el 16 de noviembre de 2012 y el 25 de enero de 2014 . Durante este tiempo se llevaron a cabo siete sesiones de diálogo mediante la herramienta Open Space Technology (OST), y una sesión final mediante la técnica Consenso Escalonado.

En el presente apartado del artículo se presentará la herramienta de diálogo OST utilizada durante el proceso, el colectivo de participantes implicados en el mismo, así como las etapas y los resultados producidos en los espacios de diálogo. 


\section{La herramienta de diálogo. Open Space Technology, una herramienta de diálogo autoorganizado}

Open Space Technology es una herramienta de diálogo fácil de aprender y usar, que puede ser aplicada para resolver gran cantidad de cuestiones relevantes y complejas de manera autoorganizada y efectiva, con una gran diversidad de actores con puntos de vista diferentes (Owen 1997a y 1997b). Su funcionamiento requiere de pasión y responsabilidad por el tema de interés a tratar, es decir, un interés genuino con la cuestión.

Los principios que guían el OST son cuatro. Cualquiera que venga es la persona indicada: significa que la emergencia de cuestiones puede darse en la interacción con cualquier persona o grupo de personas con las que comparte la pasión y responsabilidad sobre un tema. Lo que suceda es la único que debe suceder: indica que ningún actor debe controlar el evento, y que hay que centrarse en el aquí y ahora y en la capacidad de sorprendernos. Cuando sea que empiece es el momento apropiado y Cuando termina, termina: señalan al reloj como un colaborador del evento que no debe marcar los tiempos de manera rígida, empezando y terminando los espacios de diálogo cuando los grupos dispuestos al efecto lo decidan.

El elemento central y distintivo del OST como Método de Diálogo con Grandes Grupos es la autoorganización (Bunker y Alban, 2006). El propio colectivo de participantes decide los temas a tratar y su orden en la agenda, y cada participante decide en qué espacios quiere participar, sobre qué temas quiere dialogar; por lo tanto, la formación de los grupos de diálogo se genera de manera espontánea y autoorganizada partiendo de los intereses de cada participante.

Una particularidad del OST, su única ley, la Ley de Los Dos Pies, dice así: "Si durante nuestro tiempo juntos, te encuentras en una situación en la que ni aprendes ni contribuyes, usa tus dos pies y vete a un lugar más productivo" (Owen, 2008). Esta herramienta permite que las personas puedan cambiar de espacio de diálogo en cualquier momento, estar en donde más pueden aprender o aportar y donde más pueden construir conjuntamente.

Los elementos básicos de la estructura del OST son el Círculo, el Tablón de Anuncios y el Mercado. El grupo se coloca en un gran círculo para empezar a trabajar. El Tablón de Anuncios no es otra cosa que una pared o panel grande donde los participantes colocan sus asuntos de interés. En función de los asuntos de interés planteados, se crea la agenda de temas a tratar, indicándose cuándo y en qué lugar del espacio se van a abordar. 
Una vez construida la agenda, se abre el Mercado. Esto significa que todos los participantes pueden acudir a adquirir aquello que les interesa, es decir, se pueden unir a los proponentes de los temas y crear grupos para el diálogo. Cuando los primeros grupos terminan de dialogar sobre sus aspectos de interés, se abre de nuevo el Mercado, y los participantes pueden generar nuevos grupos de diálogo para conversar sobre los nuevos temas de la agenda.

Un evento OST se puede cerrar de diferentes maneras, pero una forma usual es haciendo uso del Bastón que Habla. Disponiéndose la totalidad del grupo en círculo, los participantes expresan de manera libre sus visiones, asunciones, conclusiones, reflexiones o sentimientos acerca del evento, siendo el portador del Bastón que Habla el único que puede hablar.

\section{Participantes. Un grupo multiactor}

Fueron dos los criterios establecidos para la conformación de un grupo de diálogo multiactor, plural y heterogéneo: a) la participación de personas de diferentes ámbitos de actuación en el País Vasco, principalmente el político, el social vinculado con la paz y los derechos humanos, y el académico; b) la participación a título individual y voluntaria, no en calidad de representantes de su organización de referencia.

El grupo de diálogo inicial estuvo compuesto por 22 personas -10 mujeres y 12 hombres- de entre 27 y 40 años, con una media de edad de 34. En el transcurso del proceso, el número de integrantes del grupo se fue reduciendo, ya que nuevas obligaciones laborales les impidieron seguir en el proceso de ocho sesiones de diálogo, por lo que 17 personas terminaron finalmente componiendo el grupo.

Es importante decir que no todos los participantes estuvieron presentes en las ocho sesiones de diálogo por motivos diversos (laborales, personales, de salud, etc.). Los criterios para que las sesiones de diálogo se llevaran a cabo fueron la presencia de un mínimo de 10 asistentes, y que participaran personas representativas de diferentes sensibilidades. En el apartado siguiente, el relativo al proceso de diálogo sostenido, se especificará el número de personas que participó en cada una de las sesiones de diálogo.

Del total de participantes, nueve de ellos/as pertenecen a formaciones políticas con representación en las instituciones vascas, ${ }^{2}$ por lo que todo el

2 Partido Nacionalista Vasco; Partido Socialista de Euskadi; Partido Popular de Euskadi; Unión Progreso y Democracia; Izquierda Unida; Sortu; Aralar; Alternatiba; Eusko Alkartasuna. Los cuatro últimos partidos participan electoralmente bajo el nombre de EH Bildu. 
espectro político estuvo representado. Por otro lado, cinco participantes desarrollan su trabajo en organizaciones pacifistas y de derechos humanos pertenecientes al movimiento vasco por la paz; dos de estas personas pertenecen a una organización cercana a las víctimas de ETA.

En relación con el resto del colectivo, tres son profesores universitarios de ciencias sociales y humanidades, dos provienen del ámbito sindical -siendo una de un sindicato nacionalista vasco y la otra no-, una persona es psicóloga social y organizacional, otra periodista, y otra, un ciudadano cuya madre fue amenazada por ETA, debido a su actividad política. Por último, cabe señalar que dos personas del ámbito académico y una del movimiento pacifista están vinculadas con movimientos cristianos de base.

Para elegir la muestra se utilizaron diferentes vías. En primer lugar, se contactó con los grupos de jóvenes de las distintas organizaciones políticas, se solicitó una reunión con ellas para explicarles los aspectos del proyecto -fundamentalmente la idea del OST continuado, el empoderamiento del colectivo y la multilateralidad-, y ofrecer la invitación formal para que un miembro del mismo acudiese a participar a título individual, no en calidad de representante de la organización política. En segundo lugar, se realizó igual procedimiento con cinco de las organizaciones más importantes del movimiento vasco por la paz. El resto de personas que conformaron el grupo eran conocidas por el investigador, ya que habían participado en alguna investigación previa.

\section{Etapas del proceso. Open Space Tecnology Sostenido}

El proceso de diálogo continuo no estaba diseñado de manera detallada antes de iniciarse, solamente se propuso el uso del OST como herramienta para dialogar sobre la paz en el País Vasco. El colectivo de participantes fue quien creó la agenda de temas a tratar. Ésta se creó mediante la estrategia del Tablón de Anuncios propia del OST. Tras proponer todos los temas de interés para los/as participantes, se crearon siete grandes bloques de cuestiones; el equipo de investigación propuso el calendario concreto del proceso.

Un aspecto importante del proceso fue la devolución sistemática de la información. En cada sesión de diálogo, el colectivo recogió actas donde se recopilaron las ideas, conocimientos y visiones sobre los diferentes temas tratados, además de acuerdos que se iban alcanzando. Las actas fueron recogidas y transcritas de manera literal, generándose un documento de actas con las principales aportaciones de cada sesión de diálogo. En total, se recogieron algo más de 100 páginas de ideas sobre los diferentes temas tratados. Cada 
documento de actas fue enviado vía correo electrónico al colectivo para que cada participante pudiera analizarlo y realizar nuevas aportaciones complementarias si así lo consideraba.

El proceso tuvo ocho etapas, una sesión inicial para establecer el calendario de temas a tratar, seis sesiones de diálogo en OST para abordar las diferentes cuestiones planteadas, y una sesión final para establecer consensos básicos.

1. Sesión Inaugural. Construcción de un calendario de sesiones de diálogo y dinámica de diálogo apreciativo

En la primera sesión de diálogo participaron 16 miembros del grupo. Tras la introducción del proceso, se llevó a cabo un ejercicio para detallar los temas a tratar durante el proceso de diálogo continuado, siguiendo el formato del Tablón de Anuncios característico del OST. Se invitó al grupo a que expusiera los retos principales que plantea la construcción de la paz en el País Vasco. Una vez anunciados todos los temas, éstos fueron unidos por afinidad, creándose siete grandes bloques. Tras ello, se realizó una priorización de los temas mediante una votación ponderada -los participantes otorgaban siete puntos al bloque que consideraban que debería tratarse en primer lugar, seis puntos al segundo, y así sucesivamente hasta llegar al bloque que entendieran debía abordarse en último lugar, al que otorgarían un punto-. El orden de los bloques de temas que siguió el proceso fue:

a) Paz, conflicto y violencia

b) Memoria y relato histórico

c) Cuestiones políticas

d) Participación ciudadana para la paz

e) Reconciliación y convivencia los presos

f) Reparación integral de las víctimas; Tratamiento de la cuestión de

Para terminar la sesión inicial, se planteó un ejercicio de diálogo apreciativo con tal de abordar los aspectos positivos de los que dispone la sociedad vasca para alcanzar la paz. En grupos de cuatro, los participantes fueron planteando y dialogando sobre las características de la sociedad que pueden servir para el desarrollo del proceso y la creación de un escenario de paz sostenible en el País Vasco.

2. Sesión temática: a) Paz, conflicto y violencia

En la primera sesión de diálogo del calendario participaron 20 personas. Se abordaron temáticas vinculadas con la paz, el conflicto y la violencia, tanto a nivel general o abstracto como asociadas al conflicto vasco.

El Tablón de Anuncios se compuso de 23 cuestiones de interés, que fueron estructurados en seis espacios de diálogo. Dos espacios fueron dedicados a la 
paz, uno de ellos para hablar del concepto de paz a nivel abstracto y global, y otro vinculado con la paz en el contexto vasco. De igual manera, se crearon dos espacios dedicados al conflicto, uno de carácter general y otro sobre el caso vasco, y dos espacios donde se dialogó acerca de la violencia: el primero dirigido a las diferentes formas y conceptos de violencia, y un segundo sobre las diferentes formas de violencia y perpetradores de la misma en el contexto vasco.

\section{Sesión temática: b) Memoria y relato histórico}

En la segunda sesión de diálogo estuvieron presentes 15 participantes. En esta sesión se abordaron cuestiones relativas a la construcción de la memoria y el relato histórico del pasado.

El Tablón de Anuncios estuvo compuesto por 32 cuestiones de interés que fueron unidas por afinidad para ser tratadas en seis espacios de trabajo. Dos de ellos estuvieron dedicados a la construcción de un relato común y a las políticas públicas de memoria. Un tercer espacio, a las vulneraciones de derechos humanos, las víctimas, las motivaciones de los diferentes perpetradores, y al sentido ético y político de la violencia. En el cuarto espacio se dialogó acerca del papel de los partidos políticos y agentes sociales ante el conflicto y la violencia. El quinto estuvo dedicado al papel de la participación ciudadana en la construcción de la memoria y el relato histórico. Por último, en el sexto espacio se abordó la transmisión de la memoria y el relato histórico a las nuevas generaciones.

\section{Sesión temática: c) Cuestiones politicas}

En el tercer momento participaron 10 personas, y se abordaron cuestiones relativas a la dimensión política del conflicto vasco.

Se propusieron 14 cuestiones de interés en el Tablón de Anuncios, posteriormente estructuradas en cuatro espacios de diálogo. En el primer espacio se habló acerca de la importancia de las cuestiones sociales en la construcción y desarrollo de los Estados nacionales. En el segundo se trató el tema del nacionalismo, las diferentes formas de nacionalismo y de construcción nacional. En un tercer espacio se dialogó sobre la necesidad de poner el objetivo de la paz por encima de los intereses partidistas para poder llegar a acuerdos específicos. En el último espacio se habló acerca de la legitimidad de decidir el futuro del estatus político del País Vasco de forma democrática y legal.

\section{Sesión temática: d) Participación ciudadana para la paz}

En este espacio participaron 10 miembros del grupo, y se dialogó acerca del papel de la ciudadanía en el desarrollo del proceso de paz.

Tras proponer 15 cuestiones de interés, se crearon tres espacios de diálogo. Un primer espacio estuvo dedicado al para qué de la participación ciudadana, 
es decir, al objetivo que debe cumplir la participación de la ciudadanía para la paz, y sobre qué ámbitos debería participar la ciudadanía. En segundo lugar, se habló sobre cómo facilitar que la ciudadanía participe en el proceso de paz, sobre qué herramientas deberían disponerse para su participación. En último lugar, se dialogó sobre los diferentes sectores sociales que deberían tener una participación activa en la construcción de la paz.

6. Sesión temática: e) Reconciliación y convivencia

En esta sesión participaron nuevamente 10 personas, y se trabajó sobre diferentes cuestiones vinculadas con la reconciliación social y la reconstrucción de la convivencia ciudadana.

Se propusieron 12 nuevas cuestiones para ser tratadas y se crearon cuatro espacios de diálogo. En el primero se dialogó acerca de las implicaciones directas de la reconciliación social, como por ejemplo el papel del perdón y el reconocimiento del daño causado, o la capacidad de gestionar la memoria y el olvido social.

El segundo se centró en la posibilidad de aprender de procesos de paz y reconciliación que se han producido en otros contextos, así como de los agentes del ámbito vasco e internacional que pueden contribuir a la reconciliación. En el tercero se conversó sobre la situación actual del proceso de reconciliación y sobre la importancia de la dimensión social para la reconciliación; y por último, en el cuarto espacio se habló de la importancia de la simbología y la cuestión lingüística para la convivencia identitaria en el País Vasco, así como de la relevancia de la mediación y la influencia de las negociaciones políticas en la reconciliación.

7. Sesión temática: f) Victimas y presos

En la última sesión de cuestiones de la agenda estuvieron presentes 13 miembros del grupo. En esta sesión se trataron cuestiones vinculadas con las víctimas del conflicto y con la situación de los presos de ETA y el MLNV.

Se plantearon 15 nuevos temas y se estructuraron cuatro espacios de diálogo. El primero de ellos estuvo dedicado a hablar sobre cuál debe ser la definición de víctima desde dónde partir, el papel de los colectivos de víctimas y presos en distintos ámbitos del proceso de paz, y el papel de la sociedad y de ETA para con las víctimas y los presos. El segundo espacio se centró en cómo visibilizar, reconocer y reparar a las víctimas, en las consecuencias legales, sociales y económicas de ser víctima o preso, así como el posible uso del dolor compartido como punto de encuentro entre víctimas.

Un tercer espacio estuvo dedicado a dialogar acerca del papel del perdón para la reconciliación, y de la posibilidad de colaborar por parte de los presos en dicha reconciliación, así como del papel que pueden tener los encuentros 
individuales entre víctima y victimario - presos y víctimas de ETA- que se han producido y que pueden producirse en el futuro. En el cuarto espacio de diálogo se habló sobre las fórmulas para la reinserción de los presos, sobre cómo tratar la cuestión de los presos a los que no se ha aplicado la doctrina Parot, las consecuencias de la derogación de esa doctrina por parte del Tribunal Europeo de Derechos Humanos (ECHR, 2013); ${ }^{3}$ asimismo, se dialogó acerca de la igualdad o no ante la justicia y el cumplimiento diferencial de penas por parte de diferentes victimarios. ${ }^{4}$

\section{Sesión final. Construcción de consensos básicos}

En la sesión de diálogo final no se usó el OST, sino que se desarrolló con base en otra herramienta de diálogo, pues el objetivo era lograr una serie de consensos básicos sobre todos los temas tratados durante las sesiones temáticas. La herramienta que se ocupó fue el Consenso Escalonado o Consenso por Inundación.

Esta herramienta consiste en distribuir el grupo en parejas. Cada miembro de la pareja presenta sus propuestas sobre las cuestiones que deben ser consensuadas; una vez realizado este paso, las parejas llegan a consensos y establecen una serie de propuestas aceptadas por ambos. Cuando las parejas tienen una serie de propuestas, cada una se junta con otra pareja formando un grupo de cuatro participantes.

En este nuevo espacio, cada pareja presenta sus propuestas, se comparan y se acuerdan qué propuestas son comúnmente aceptadas. Este proceso se repite hasta que todos los participantes se reúnen en el gran grupo, y consensúan las propuestas finales. En un principio, es positivo acordar el número de propuestas que debe consensuar cada pareja, grupo de cuatro, ocho, etc.; por ejemplo, pueden ser tres propuestas por cuestión a ser consensuada.

En esta última sesión del proceso participaron 11 personas, por lo que se formaron cuatro parejas y un trío en el primer paso, un grupo de cinco y otro de seis en el segundo, para finalmente terminar todo el grupo en un mismo espacio.

3 Jurisprudencia establecida por el Tribunal Supremo de España el 28 de febrero de 2006, conocida con la Doctrina Parot, que permite la acumulación de penas y la permanencia ilimitada de presos en centros carcelarios. La sentencia del Tribunal Europeo de Derechos Humanos anula la Doctrina Parot, al entender que vulnera el artículo 7 del Convenio de Derechos Humanos de la Unión Europea.

4 Victimarios pertenecientes a GAL, Triple A, Batallón Vasco-Español o a los Cuerpos y Fuerzas de Seguridad del Estado han recibido un trato favorable en materia penitenciaria a través de indultos o reducción de penas. 
Como se ha mencionado anteriormente, la dinámica de diálogo estuvo centrada en consensuar aspectos básicos de todas las cuestiones tratadas durante el calendario de sesiones. Para ello, en el momento inicial de la sesión se entregó a cada participante un documento donde se resumían todas las actas de los espacios de diálogo, y otro documento donde aparecían las cuestiones que debían ser consensuadas. Luego se dio un tiempo de 30 minutos para que cada participante pudiera madurar sus propuestas en relación con cada una de las cuestiones que debían ser consensuadas. Una vez realizada la maduración individual, se procedió al trabajo de consenso por parejas, posteriormente se llevó a cabo el consenso en dos grupos de cinco y seis personas, para finalmente establecer el consenso final en el grupo.

Las cuestiones a consensuar fueron divididas en seis bloques, con tres cuestiones específicas para cada uno de ellos:

1. Reconciliación y convivencia. a) ¿Qué implica la reconciliación? Los elementos fundamentales para la reconciliación. b) ¿Cómo facilitar la reconciliación? Aspectos sobre cómo debe facilitarse la reconciliación. c) ¿Quién debe implicarse/liderar la reconciliación? Qué agentes deben implicarse en la reconciliación y quiénes deben liderarla.

2. Memoria y relato histórico. a) ¿Con qué objetivos debe construirse un relato histórico? Motivos de la construcción del relato. b) ¿Cómo se debe llevar a cabo el relato histórico? Fórmulas para construir el relato. c) ¿Qué debe incluir el relato? Cuestiones que deben aparecer en el relato histórico.

3. Dimensión política del conflicto. a) ¿Cómo dar salida al conflicto de intereses nacionalistas? Fórmulas para avanzar en el logro de acuerdos. b) ¿Existen fórmulas que puedan satisfacer a todas las partes? Posibles acuerdos en el que todas las partes puedan tener cabida. c) ¿Cómo dar salida al conflicto de identidades? Elementos para construir un escenario de convivencia identitaria.

4. Participación ciudadana. a) ¿Qué objetivos debe tener la participación ciudadana? El para qué de la participación ciudadana. b) ¿Qué rol debe tener la ciudadanía? Ámbitos y aspectos donde debe participar la ciudadanía. c) ¿Cómo llevar a cabo la participación ciudadana? Fórmulas para facilitar la participación ciudadana.

5. Víctimas. a) ¿Cómo definimos a una víctima? Aspectos clave para definir a las víctimas. b) ¿Qué rol pueden jugar las víctimas en un proceso de reconciliación? Cuestiones/acciones que podrían hacer las víctimas para facilitar la reconciliación. c) ¿Cómo reparar a las víctimas? Elementos para reparar a las víctimas. 
6. Presos. a) ¿Cómo dar salida a la cuestión de los presos? Elementos para tratar la situación de los presos. b) ¿Cómo facilitar la reinserción? Elementos para favorecer la reinserción de los presos. c) ¿Qué rol pueden jugar los presos en un proceso de reconciliación? Cuestiones/acciones que podrían hacer los presos para facilitar la reconciliación.

\section{Análisis de los datos}

Los datos del estudio que se exponen en el presente artículo hacen referencia a los asuntos tratados y acuerdos alcanzados en las diferentes sesiones de diálogo, especialmente los consensos establecidos durante la última sesión. El análisis de las actas se realizó mediante la creación de categorías emergentes sobre las diferentes cuestiones tratadas; posteriormente se llevó a cabo un contraste con el colectivo de participantes para la confirmación de los consensos alcanzados, y tras ello, la creación de un informe final de conclusiones sobre el proceso realizado.

\section{Resultados. Ideas y consensos para la construcción de paz en el País Vasco}

El objetivo básico del trabajo era establecer una serie de consensos básicos sobre todas las cuestiones planteadas; por este motivo, en el presente apartado se van a presentar los consensos logrados tanto en las distintas sesiones de diálogo, como, sobre todo, en la sesión final. Siguiendo este orden, se presentarán acuerdos e ideas relativos a: 1) implicaciones de la paz, la reconciliación y la convivencia; 2) la memoria y el relato histórico; 3) la dimensión política del conflicto; 4) la participación ciudadana para la paz; 5) las víctimas; 6) los presos.

\section{Implicaciones de la paz, la reconciliación y la convivencia}

El colectivo de participantes señala que la paz es un fenómeno polisémico, siendo a la vez proceso y el fin último de la sociedad. Se requiere de espacios y de pasos sostenidos para construirla, es imprescindible superar las diferentes formas de violencia y la vulneración de derechos humanos presentes en el conflicto vasco. Se apunta que el desarme de ETA y producir cambios en la política antiterrorista y penitenciaria son elementos que facilitarían los distintos aspectos que implica la construcción de la paz.

Por otro lado, un escenario de paz sostenible se vincula al desarrollo de una cultura democrática y de paz construida desde la tolerancia, el respeto y la empatía hacia el diferente, permitiendo crear nuevos sentidos de la vida 
distintos a los construidos durante el conflicto. La reconciliación se afirma como un proceso global que afecta a toda la sociedad y que implica la transformación de las prácticas sociales. Para construir un marco de convivencia sostenible, se indica la necesidad de apostar por el futuro en común, pero manteniendo viva una memoria social crítica de lo sucedido. Se señala la necesidad de crear espacios formales e informales para el diálogo ciudadano desde el cual abordar el reto de transformar las prácticas sociales propias del conflicto en prácticas sociales de convivencia.

Un elemento a destacar es el reconocimiento de las responsabilidades sobre el sufrimiento y el daño causado. Se indica que el reconocimiento debe realizarse desde una perspectiva ética, con honestidad y contenido, y desde la autocrítica por haber realizado vulneraciones a los derechos humanos. Vinculado con ello, se menciona la necesidad de de-construir los discursos legitimadores de violencia producidos durante el conflicto, promover el rechazo a la violencia; para ello es importante no reconocer socialmente de manera positiva acciones violentas.

Un aspecto trascendental versa sobre quién debe implicarse y liderar la reconciliación. El colectivo indica que el liderazgo debe ser compartido de manera horizontal entre las instituciones y administraciones públicas, los partidos políticos, los agentes de la sociedad civil organizada, el ámbito educativo, etc., implicando a la sociedad en su conjunto. Se pone el énfasis en la responsabilidad de los partidos políticos para la reconciliación, siendo positivo sacar el tema de la paz de la confrontación política, utilizando estilos comunicativos mesurados y actitudes conciliadoras y empáticas con el diferente, aunque sin renunciar a expresar las posiciones propias con asertividad.

En los documentos también se aprecia la importancia de resaltar lo positivo de la sociedad vasca donde se han dado experiencias civiles e institucionales favorables a la paz y la reconciliación.

\section{La memoria y el relato histórico}

El papel de la memoria y el relato histórico para la construcción de la paz y la reconciliación en el País Vasco aparece vinculado con varias cuestiones. En primer lugar, los objetivos del trabajo por la memoria y el relato histórico deben incluir la comprensión del pasado para superar el conflicto violento, garantizar la no repetición de los hechos, la construcción de un marco de convivencia basado en el respeto a los derechos humanos, facilitar la de-construcción de las lógicas legitimadoras de la violencia, y reconocer las vulneraciones a los derechos humanos cometidas para poder construir la verdad necesaria en un proceso de reconocimiento social y de reparación integral de las víctimas. 
Respecto a la memoria, un primer aspecto es la necesidad de no hacer un uso partidista de ésta desde los diferentes sectores sociales y políticos. En términos de procedimiento, se apunta la posibilidad de realizar un proceso participativo-inclusivo, donde todas las visiones y vivencias sobre el conflicto sean recogidas a partir de espacios de diálogo y recopilación de testimonios. Complementario a ello, se plantea la importancia de llevar a cabo un relato de hechos objetivos, especialmente aquellos que están vinculados con vulneraciones de derechos humanos, principalmente el derecho a la vida y a la integridad física, psicológica y moral.

Por otro lado, ante la existencia de diferentes relatos subjetivos sobre el pasado, se evidencia la necesidad de reconocer que existen distintas visiones del pasado, pero generando cauces para posibilitar un acuerdo mínimo entre los diferentes sectores con visiones distintas, es decir, posibilitando el desarrollo de memorias más inclusivas.

Un tercer aspecto se refiere a los contenidos que debe incluir el relato de lo sucedido. Estableciéndose como punto de partida el respeto a los derechos humanos, se indica la necesidad de establecer una relación verídica de todas las vulneraciones. Obtener una fotografía completa de las víctimas, sin establecer comparaciones ni jerarquías sobre sus procesos de victimización, puede facilitar la elaboración de consensos sobre la interpretación del pasado.

En esta interpretación tienen cabida tanto los hechos objetivos, como las vivencias y sentimientos de las personas que aportan sus testimonios. Una última cuestión se refiere a la necesidad de que el relato incluya análisis sobre los obstáculos que los diferentes agentes del conflicto hayan podido interponer en la búsqueda de la convivencia pacífica, así como las experiencias que la han favorecido.

\section{La dimensión politica del conflicto}

Dos elementos importantes para comprender el conflicto político, y por ende, para buscar su transformación, son la territorialidad y el sujeto político de decisión. Podríamos decir que el núcleo del conflicto político es la posibilidad de ejercer el derecho de autodeterminación, y qué territorios pueden ejercer dicho derecho.

Para dar salida al conflicto, el colectivo de participantes percibe como imposible que los distintos sectores lleguen a un acuerdo sobre la definición del sujeto político y por lo tanto, sobre el derecho de autodeterminación. A pesar de ello, para encontrar fórmulas que puedan satisfacer a todas las partes, se indica que las soluciones tienen que pasar por el desarrollo de fórmulas inclusivas desde las variables de identidad y participación, y por consensuar procedimientos, para poder preguntar a la ciudadanía acerca de su voluntad política. 
A nivel identitario, la interdependencia negativa entre las identidades colectivas vasca y española es uno de los elementos centrales del conflicto vasco. Ante este hecho, se señala que la transformación del conflicto de identidades requiere de un reconocimiento de la pluralidad identitaria presente en la realidad vasca para su protección, sobre todo en lo lingüístico, indicando que los grupos identitarios no podrán conseguir el 100\% de sus objetivos en el marco de una solución global. De igual modo, se apuesta por separar el conflicto de identidades de la dimensión violenta del conflicto, ya que la defensa de las identidades colectivas no está sujeta al uso de la violencia.

Un último aspecto que indica el colectivo, y que puede ser una premisa para estructurar nuevas formas de convivencia política en el País Vasco, es desarrollar visiones dinámicas de la historia, donde las situaciones políticas cambian y pueden ser cambiadas de manera democrática con el objetivo de vivir mejor.

\section{Participación ciudadana para la paz}

La participación ciudadana se ha considerado como un elemento esencial para la conformación de un escenario de paz sostenible. Los resultados indican que la participación ciudadana para la paz tiene la finalidad de otorgar un protagonismo directo a la ciudadanía en todas las cuestiones que entraña el proceso de paz. Se incide en la importancia de establecer relaciones de influencia bidireccional entre los estratos elevados de toma de decisiones -instituciones, partidos políticos, etc.- y la sociedad civil. Para ello, se señala la relevancia de empoderar a la sociedad, estableciendo foros sociales y otros mecanismos de participación directa. Un aspecto importante al respecto es la necesidad de que la ciudadanía participe en espacios plurales y en foros creados por los rivales o enemigos políticos, no sólo en aquellos organizados por el propio colectivo, traspasando así las barreras del conflicto.

En cuanto a los roles que debe llevar a cabo la ciudadanía vasca, se indica que, por un lado, debe ejercer un rol reflexivo para aportar ideas que puedan ayudar a avanzar, y, por otro, ejercer un rol participativo directo y de "lobby social" para presionar en la solución de los problemas.

Los documentos señalan que la participación ciudadana debe llevarse a cabo mediante fórmulas de distinta índole. Se hace énfasis en los espacios de diálogo ciudadano con representación plural y heterogénea, y en generar cauces para que las decisiones tomadas en el seno de la ciudadana tengan carácter vinculante. Asimismo, se apunta la importancia de las estructuras intermedias de la sociedad, y del trabajo a nivel local, ya que son espacios con posibilidad de crear alternativas y soluciones para la reconciliación y la reconstrucción del tejido social. 


\section{Las victimas}

Un aspecto imprescindible para consensuar el papel que deben tener las víctimas en la construcción de paz, así como para proponer cuestiones sobre la reparación integral, es compartir una idea o definición de víctima. Esta ha sido una cuestión importante en el caso del conflicto vasco, presentándose grandes dificultades para reconocer el estatus de víctima a diferentes víctimas del conflicto, pues implica la presencia de distintos perpetradores.

En el proceso desarrollado, el colectivo de participantes entiende que las víctimas son aquellas personas que han sufrido vulneraciones de los derechos humanos y un daño inmerecido por parte de los diferentes agentes perpetradores de violencia directa (ETA y grupos afines, Fuerzas Policiales, GAL, y grupos de extrema derecha [Batallón Vasco Español, Triple A]). Además, se asume que las víctimas del conflicto son aquellas que han sufrido violencia política asociada al conflicto vasco, y que por su calidad de víctima merecen reparación.

Los resultados apuntan a la necesidad de establecer una clasificación de víctimas en función de las vulneraciones de derechos humanos sufridas, pero sin que ello suponga una fuente de comparación entre ellas. Además, en dicha clasificación de víctimas cabe la posibilidad de introducir la figura de víctima/victimario, pues existen casos de personas que han sido perpetradores de violencia y han cometido vulneraciones a los derechos humanos, y al mismo tiempo han sido víctimas.

En cuanto al rol que pueden jugar las víctimas para facilitar un proceso de reconciliación, se afirma lo positivo de brindar testimonio de sus experiencias de manera voluntaria y sin intencionalidad política, o de generar espacios de encuentro entre diferentes víctimas donde se facilite la empatía, la humanización y el perdón, siempre desde la voluntariedad, el respeto y la discreción. Estos dos casos aparecen muy vinculados con la necesidad de transmitir imágenes positivas y ejemplarizantes a la sociedad.

De igual modo, se enfatiza el requerimiento de un reconocimiento social e institucional que favorezca su visibilización y reparación exento de manipulaciones del sufrimiento por parte de agentes políticos y sociales, y se apunta el reconocimiento mutuo del sufrimiento entre víctimas de diferentes perpetradores como un aspecto importante para la reconciliación entre ellas.

Para reparar a las víctimas, se pone de relieve la necesidad de su reconocimiento a nivel social e institucional, y garantizar sus derechos a la verdad, la justicia, la reparación y la no repetición, enfatizándose que los crímenes recibidos no queden impunes. También se señala la importancia de devolver un estatus de "normalidad" a la vida cotidiana de las víctimas, no realzando 
constantemente su rol de víctima, y no requiriendo de ellas para cualquier aspecto vinculado con la paz y la reconciliación, ya que ello puede re-traumatizarlas, haciendo que sus roles sociales siempre estén relacionados con su victimización o la de sus familiares.

\section{Los/las presos}

La temática de los presos vinculados con ETA y con el MLNV es una cuestión central para la construcción de paz en el País Vasco. Las directrices de la política antiterrorista del Estado español han supuesto un tratamiento excepcional a las personas de ETA o el MLNV encarceladas, lo que se ejemplifica en la política de dispersión de presos, ${ }^{5}$ y en la no aplicación de medidas penitenciarias aplicables a cualquier recluso.

Se apunta a la necesidad de un proceso bilateral entre los presos de ETA y el Estado, indicándose que el gobierno del Estado Español tiene que responder a los pasos dados por el colectivo de presos de ETA, como por ejemplo la aceptación por parte de éstos de la legalidad penitenciaria española.

En términos concretos, los resultados afirman la importancia de acabar con la dispersión de los presos, acercándolos a las cárceles próximas a sus domicilios, de excarcelar a presos enfermos, favorecer salidas individualizadas mediante la aplicación de la legislación penal ordinaria, y revisar las penas en función de los tipos de crímenes cometidos (asesinatos, kale borroka, ${ }^{6}$ política, ${ }^{7}$ etc.). A estas cuestiones se añade la necesidad de facilitar canales reales de reinserción por parte de las administraciones públicas competentes, entendiéndose que la reinserción pasa por no poner penas extras a los expresos negándoles derechos civiles o políticos o favoreciéndose la inserción laboral.

Por último, se señala que los presos pueden tener un rol activo en el establecimiento de un escenario de paz sostenible. Se indica que esto puede estar asociado a una renuncia expresa de la violencia para favorecer su deslegitimación social, a aceptar el estado de las vulneraciones cometidas colaborando con su esclarecimiento, o a aportar imágenes positivas para la reconciliación y realizando un trabajo pedagógico para la sociedad en general y para los afines ideológicamente. Además se afirma que la salida de los presos debe llevarse a cabo con discreción y mesura, de tal manera que no suponga un agravio a las víctimas.

5 La política de dispersión de los presos de ETA, que comienza en el año 1989, supone que éstos no cumplen sus condenas en las cárceles próximas a sus domicilios, sino en otras cárceles españolas.

6 La Kale Borroka es el término acuñado para designar la violencia callejera empleada por grupos pertenecientes al MLNV.

7 Pertenencia a partidos políticos o agentes sociales del MLNV, declarados como pertenecientes al entramado de ETA. 


\section{Reflexiones finales}

Tal y como afirma Jonhson (2003), la complejidad y la autoorganización han pasado de ser una forma de entender la realidad a una forma de construirla. El presente estudio para la paz en el País Vasco es un pequeño ejemplo de puesta en práctica del enfoque sistémico y complejo en transformación de conflictos y construcción de paz. En este sentido, siguiendo a Wills y colaboradores (Unger y Wills, 2006; Wills et al., 2006) y a Coleman y colaboradores (Coleman, 2006 y 2012; Coleman et al., 2007), el proceso de diálogo generado ha seguido las premisas de multiparcialidad e inclusividad, haciendo partícipe a personas de diferentes agentes claves del conflicto, y a la vez claves para la paz, como son miembros de partidos políticos con vocación de avanzar en la transformación de la situación en el País Vasco, personas del movimiento vasco por la paz o académicos implicados con la cuestión.

De igual modo, se ha puesto en práctica un ejercicio de diálogo sostenido basado en la autoorganización, el empoderamiento colectivo y la democracia deliberativa, siendo éstos valores y prácticas centrales del enfoque sistémico y complejo de la transformación de conflictos, e imprescindibles para la emergencia de nuevas ideas y alternativas para la paz.

Durante el transcurso de los encuentros se fueron construyendo relaciones de confianza entre las y los participantes, se hizo más compleja la visión de los otros, superándose estereotipos y prejuicios previos. Esto permitió la práctica del diálogo intenso y profundo sobre las cuestiones más relevantes del conflicto vasco y los retos más importantes para lograr un escenario de paz sostenible. Finalmente, se hizo realidad la posibilidad de llegar a acuerdos básicos entre personas con diferentes visiones, intereses, necesidades y valores vinculados al conflicto y la paz en el País Vasco.

En cuanto al contenido de los consensos construidos y su alcance, y partiendo de una visión sistémica del conflicto vasco y la construcción de paz, se puede observar cómo el colectivo de participantes ha puesto énfasis en la transformación de atractores del conflicto mencionados en la descripción del caso de estudio (Mínguez-Alcaide, 2013), y ha aportado posibles atractores de paz para construir un escenario de paz sostenible.

Aunque de manera no muy extensa y exhaustiva, las recomendaciones aportan una visión integral de la construcción de la paz en el País Vasco, desde un enfoque amplio y procesual de la reconciliación y el desarrollo de la convivencia, una paz con múltiples dimensiones que deben ser abordadas desde la multiparcialidad y la búsqueda del bien común. 
En concreto, y haciendo un viaje por la transformación de los atractores del conflicto y la creación de atractores de paz, se explicita la necesidad de finalizar con las expresiones y relaciones basadas en la violencia -atractor del conflicto-, a partir del desarme de ETA y el cambio en la política antiterrorista y penitenciaria del Estado español, y la creación de un marco, unos procedimientos y unas prácticas basadas en los derechos humanos para la reparación integral de las víctimas y el desarrollo de una salida a la cuestión de los presos -atractor de paz-.

Por otro lado, a lo largo de los consensos alcanzados se pone de manifiesto la importancia de romper con los discursos legitimadores de la violencia -atractor del conflicto-, y de-contruir esos discursos a partir de la autocrítica y el reconocimiento del daño injusto causado a las víctimas por parte de los actores armados del MLNV, los agentes del Estado español, y grupos parapoliciales y de extrema derecha, el reconocimiento social, institucional y político de las vulneraciones a los derechos humanos, y el desarrollo de medidas efectivas de verdad, justicia y reparación -atractor de paz-.

En cuanto al anclaje político e identitario del conflicto, la superación de la contradicción nacional y la interdependencia negativa de las identidades vasca y española -atractores del conflicto-, se plantea desde el reconocimiento de la pluralidad identitaria y la importancia de satisfacer el desarrollo de las identidades colectivas, a la par que se apunta hacia la profundización democrática como idea para generar fórmulas políticas que puedan satisfacer a la mayoría de la sociedad vasca - atractores de paz-.

Además de ello, se considera importante destacar la recomendación aportada por el colectivo relativa a favorecer el liderazgo compartido para la paz entre todos los estamentos de la sociedad vasca, desde la participación plural y heterogénea como elemento transversal en el abordaje de las distintas dimensiones de la paz, la construcción de relato y la memoria, y la reparación integral de las víctimas, hasta la contradicción nacional definitoria de la dimensión política del conflicto.

Los resultados afirman que, a pesar de la responsabilidad de las instituciones y partidos políticos, el liderazgo del proceso debe ser compartido por todos los agentes de la sociedad vasca. En este sentido, se recomienda transitar hacia un escenario de paz haciendo que la ciudadanía tenga un activo y central del cambio, creando espacios de diálogo donde transformar prácticas sociales marcadas por la división y la desconfianza en marcos de convivencia social. 
La centralidad de la participación y el diálogo ciudadanos son especialmente relevantes en la transformación del sustrato psicosocial del conflicto, es decir, las percepciones simplificadas, distorsionadas y polarizadas de la realidad, las imágenes del otro, las actitudes con las cuales se ha afrontado el conflicto, las emociones que despierta el conflicto o los procesos morales de exclusión y deshumanización. En este sentido, se aporta la necesidad de diálogo para hacer emerger nuevos atractores psicosociales para la paz, como el desarrollo de narrativas históricas del conflicto más complejas y menos confrontadas, de visiones más amplias de los grupos calificados históricamente como enemigos, o de la empatía desde su vertiente más cognitiva a la más emocional. En definitiva, nuevos anclajes psicosociales para la creación de un escenario de paz sostenible en el País Vasco.

Este ejercicio no ha sido el único proceso de diálogo sostenido que se ha producido en el País Vasco desde el anuncio del fin de la actividad armada de ETA en octubre de 2011. Diferentes organizaciones sociales han dinamizado y dinamizan espacios de diálogo con miembros de la ciudadanía, con jóvenes de los partidos políticos o con representantes públicos a nivel municipal. Estos procesos están siendo implementados de manera discreta, básicamente en municipios de la provincia de Gipuzkoa. Algunas diferencias entre estos procesos y el que se presenta en este estudio, son la naturaleza multiactor de este último, la utilización de herramientas de diálogo con grandes grupos como el OST, o su carácter académico asociado a la aproximación sistémica de la transformación de conflictos y la construcción de paz.

\section{Referencias}

Alvarez-Enparantza, José Luís (1997), Euskal Herria en el horizonte, España: Txalaparta. Apalategi, Jokin (2006), Los vascos, de la nación al estado, España: Herritar Berri.

Bunker, Barbara Benedict y Alban, Billie T. (2006), The Handbook of Large Group Methods. Creating Systemic Change in Organizations and Communities, Estados Unidos: JosseyBass Publishers.

Coleman, Peter T. (2006), "Conflict, complexity, and change: A meta-framework for addressing protracted, intractable conflicts - III", en Peace and Conflict: Journal of Peace Psychology, vol. 12, núm. 4, Estados Unidos: American Psychological Association.

Coleman, Peter T. (2012) "Conclusion: The Essence of Peace? Toward a Comprehensive and Parsimonious Model of Sustainable Peace", en Coleman, Peter T. y Deutsch, Morton [eds.], Psychological Components of Sustainable Peace, Estados Unidos: Springer.

Coleman, Peter T. et al. (2007), "Intractable conflict as an attractor: Presenting a dynamical model of conflict, escalation, and intractability, en American Behavioral Scientist, vol. 50, núm. 11, Estados Unidos: Sage Journals. 
Duran, Lauren (2013), Conflicts in Myanmar: A systemic approach to conflict analysis and transformation. Disponible en: http://lup.lub.lu.se/luur/downloadfunc=downloadFi le\&recordOId=3809452\&fileOId=3809455 [12 de junio de 2015].

European Court of Human Rights (EHCR) (2013), Sentencia de la Gran Sala de la Corte Europea de Derechos Humanos sobre la demanda número 42750/09. Asunto Del Río Prada vs. España. Disponible en: http://hudoc.echr.coe.int/sites/eng/pages/search.as px?i=001127697\#\{“itemid”:[“001-127697”]\} [12 de junio de 2014].

ETA (2011a), Comunicado de declaración de alto el fuego permanente $y$ de carácter general. Disponible en: http://estaticos.elmundo.es/ documentos/2011/01/10/comunicado_eta.pdf [08 de junio de 2011].

ETA (2011b), Comunicado de anuncio del cese definitivo de la actividad armada. Disponible en: http://gara.naiz.info/eta-anuncia-cese-definitivo-actividad-armada.php [21 de noviembre de 2011].

Gawerc, Michelle I. (2006), "Peace-Building: Theoretical and concrete perspectives", en Peace \& Change, vol. 31, núm. 4, Estados Unidos: Peace History Society.

Gurr, Ted Robert (2000), Peoples Versus States: Minorities at Risk in the New Century, Estados Unidos: United States Institute of Peace.

Horowitz, Donald L. (1985), Ethnic Groups in Conflict, Estados Unidos: University of California Press.

Johnson, Steven (2003), Sistemas emergentes. O qué tienen en común hormigas, neuronas, ciudades y software. Madrid: Turner Publicaciones.

Körpen, Daniela et al. (2011), The Non-linearity of Peace Processes: Theory and Practice of Systemic Conflict Transformation, Estados Unidos: Barbara Budrich Publishers.

Lederach, John Paul (1998), Construyendo la Paz. Reconciliación sostenible en sociedades divididas, España: Bakeaz y Gernika Gogoratuz.

Lizarralde, Imanol (2012), Teoría francesa y táctica y estrategia del MLNV (1967-2009), Universidad del País Vasco: Tesis doctoral.

Mínguez-Alcaide, Xavier (2013), Una aproximación psicosocial al conflicto vasco. Construyendo la paz en espacio abierto, Universidad del País Vasco: Tesis doctoral.

Novak, Andrzej et al. (2012), "Sustainable Peace: A Dynamical Systems Perspective”, en Coleman, Peter T. y Morton Deutsch [eds.], Psychological Components of Sustainable Peace, Estados Unidos: Springer.

Owen, Harrison (1997a), Expanding our Now: The Story of Open Space Technology, Estados Unidos: Berrett-Koehler.

Owen, Harrison (1997b), Open Space Technology: A User's Guide, Estados Unidos: BerrettKoehler.

Owen, Harrison (2008), A brief user's guide to open space technology. Disponible en: http:// openspaceworld.com/usersguide.htm [19 de septiembre de 2009].

Unger, Barabara y Wills, Oliver (2006), Systemic conflict transformation. Guiding principles for practitioners and policy makers working on conflict, Berlín: Berghof Fundation for Peace Support.

Vallacher, Robin et al. (2010), "Rethinking intractable conflict: The perspective of dynamical systems", en American Psychologist, vol. 65, núm. 4, Washington: American Psychological Association.

Wills, Oilver et al. (2006), The Systemic Approach to Conflict Transformation. Concept Fields of Application, Berlín: Berghof Fundation for Peace Support. 
Xavier Mínguez Alcaide. Doctor en Psicología por la Universidad del País Vasco. Actualmente trabaja en la Asociación Gune Irekiak como responsable del proyecto de investigación para la paz en el País Vasco: "Ehunberri: espacios y encuentros para un nuevo tejido social". Principales líneas de investigación: investigación para la paz desde una óptica psicosocial compleja, y la investigación-acción participativa. Publicaciones recientes: "Conflicto y paz en Colombia. Significados en organizaciones defensoras de los derechos humanos", en Revista de Paz y Conflictos, vol. 8 (2015); "Hacia una memoria incluyente para la paz en el País Vasco", en Política y Sociedad, vol. 52 (2015); "Una aproximación psicosocial al conflicto vasco como sistema dinámico", en Universitas Psychologica, vol. 14, núm. 2 (2015).

Recepción: 30 de junio de 2015.

Aprobación: 5 de septiembre de 2016 